PROCEEDINGS OF THE

AMERICAN MATHEMATICAL SOCIETY

Volume 131, Number 11, Pages 3511-3516

S 0002-9939(03)06958-2

Article electronically published on April 1, 2003

\title{
A MIXED TYPE SYSTEM OF THREE EQUATIONS MODELLING REACTING FLOWS
}

\author{
YUN-GUANG LU AND CHRISTIAN KLINGENBERG
}

(Communicated by Suncica Canic)

\begin{abstract}
In this paper we contrast two approaches for proving the validity of relaxation limits $\alpha \rightarrow \infty$ of systems of balance laws

$$
u_{t}+f(u)_{x}=\alpha g(u) \text {. }
$$

In one approach this is proven under some suitable stability condition; in the other approach, one adds artificial viscosity to the system

$$
u_{t}+f(u)_{x}=\alpha g(u)+\epsilon u_{x x}
$$

and lets $\alpha \rightarrow \infty$ and $\epsilon \rightarrow 0$ together with $M \alpha \leq \epsilon$ for a suitable large constant $M$. We illustrate the usefulness of this latter approach by proving the convergence of a relaxation limit for a system of mixed type, where a subcharacteristic condition is not available.
\end{abstract}

\section{Viscosity JOINING FORCES WITH RELAXATION}

In this paper we are interested in combining the zero relaxation with the zero dissipation limit of the Cauchy problem for models of reaction flow:

$$
\left.\begin{array}{rl}
v_{t}-u_{x} & =\epsilon v_{x x} \\
u_{t}-\sigma(v, s)_{x} & =\epsilon u_{x x} \\
s_{t}+c_{1} s_{x}+\frac{s-h(v)}{\tau} & =\epsilon s_{x x}
\end{array}\right\}
$$

with initial data

$$
(v, u, s)_{\mid t=0}=\left(v_{0}, u_{0}, s_{0}\right) .
$$

The third equation in (1.1) contains a relaxation mechanism with $h(v)$ as the equilibrium value for $s, \tau$ the relaxation time, $\epsilon$ the viscous parameter and $c_{1}$ a constant.

The problem (1.1) can be considered as a singular perturbation problem as $\tau$ tends to zero. When $\tau \leq \frac{\epsilon}{M}$, with $M$ a suitable large constant which depends only on the initial data, and $\epsilon$ goes to zero, the convergance of the solutions $\left(v^{\epsilon, \delta}, u^{\epsilon, \delta}, s^{\epsilon, \delta}\right)$ for the Cauchy problem (1.1), (1.2) was proven in [Lu1], Lu2 for two particular cases. In [Lu1] we consider $h(v)=v, \sigma(v, s)=\sigma(v)+s$, and in [Lu2] the case $\sigma(v, s)=c_{1} v+c_{2} s$ and $h(v)$ is a nondecreasing function. The relaxation

Received by the editors November 1, 2000 and, in revised form, June 6, 2002.

2000 Mathematics Subject Classification. Primary 35L65; Secondary 35M10.

(C)2003 American Mathematical Society 
and dissipation limit of $(v, u, s)$ in (1.1) satisfies $s=h(v)$ and $(v, u)$ is an entropy solution of the equilibrium system

$$
\left.\begin{array}{rl}
v_{t}-u_{x} & =0 \\
u_{t}-\sigma(v, h(v))_{x} & =0
\end{array}\right\}
$$

When $\epsilon=0$ and $\tau \rightarrow 0$ the relaxation limit for the Cauchy problem (1.1), (1.2) was obtained in [T] for the special case $\sigma(v, s)=c_{1} v+c_{2} s, 0<h^{\prime}(v) \leq$ c. The equilibrium solution $(v, u)$ is bounded in $L^{2}$ as is proven in [Lu2]. An important early contribution of conservation laws with relaxation [Liu introduced the subcharacteristic condition which is needed for the stability of the relaxation limit for many physical models. This condition can be illustrated with a simple example:

$$
\left.\begin{array}{rl}
v_{t}-u_{x} & =0 \\
u_{t}-c v_{x}+\frac{u-h(v)}{\tau} & =0
\end{array}\right\}
$$

The two eigenvalues for the system

$$
\left.\begin{array}{rl}
v_{t}-u_{x} & =0 \\
u_{t}-c v_{x} & =0
\end{array}\right\}
$$

are $\lambda_{1}=-\sqrt{c}, \lambda_{2}=\sqrt{c}$ and the eigenvalue of the equilibrium equation of (1.4) is $\lambda^{*}=-h^{\prime}(v)$. Then the subcharacteristic condition $(c>0)$

$$
\lambda_{1}<\lambda^{*}<\lambda_{2}
$$

will ensure the stability of solutions $\left(v^{\tau}, u^{\tau}\right)$ of (1.4) as $\tau$ goes to zero. However when $c<0$, the condition (1.6) fails to hold. In fact (1.4) is ill posed for $c<0$ as we proceed to show. By the first equation in (1.4) there must exist a function $w$ such that

$$
w_{x}=v, \quad w_{t}=u .
$$

Thus the second equation in (1.4) can be put in the form

$$
w_{t t}-c w_{x x}+\frac{1}{\tau}\left(w_{t}-h\left(w_{x}\right)\right)=0
$$

This is an elliptic equation. Its Dirichlet problem in the domain $t>0$ can be solved with the data

$$
w(x, 0)=\int_{0}^{x} v_{0}(s) d s
$$

but then $u_{0}(x)$ cannot be chosen independently of $v_{0}(x)$ since in this case we must have

$$
v_{0}(x)=w_{t}(0, x)
$$

and $w$ depands on $v_{0}(x)$. Nevertheless, if we add a viscosity to the right-hand side of (1.4)

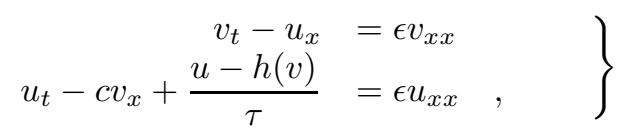

the system (1.8) is well posed. Applying the approach given in [L3] to this simple situation one can show the compactness of the viscous-relaxation approximation $\left(v^{\epsilon, \tau}, u^{\epsilon, \tau}\right)$ when $\tau \leq \frac{\epsilon}{M}, \epsilon \rightarrow 0$, for a suitable large constant $M$. 
This can be seen through an asymptotic expansion of the Chapman-Enskog type. Let

$$
u=h(v)+\tau u_{1}+O\left(\tau^{2}\right) .
$$

Then from the second equation in (1.8) we obtain

$$
\begin{aligned}
u_{1} & =\epsilon u_{x x}-u_{t}+c v_{x}+O(\tau) \\
& =\epsilon u_{x x}-h^{\prime}(v) v_{t}+c v_{x}+O(\tau) \\
& =\epsilon u_{x x}-\epsilon h^{\prime}(v) v_{x x}-h^{\prime}(v) u_{x}+c v_{x}+O(\tau) \\
& =\epsilon u_{x x}-\epsilon h^{\prime}(v) v_{x x}-h^{\prime 2}(v) v_{x}+c v_{x}+O(\tau)
\end{aligned}
$$

Substituting (1.10) into the first equation in (1.8), we get

$$
\begin{aligned}
v_{t}-h(v)_{x} & =\epsilon v_{x x}+\tau u_{1 x}+O\left(\tau^{2}\right) \\
& =\left(\left(\epsilon+\tau\left(c-h^{\prime 2}(v)\right)\right) v_{x}\right)_{x}+O\left(\tau^{2}\right)+O(\tau \epsilon)
\end{aligned}
$$

Thus if $\epsilon>\tau\left(h^{\prime 2}(v)-c\right)$, the equation (1.11) is well posed. This is also the effect of the subcharacteristic condition in (1.11) with $\epsilon=0$.

This illustrates that viscosity is not only of mathematical expedience when acting together with relaxation but may also be a necessary stability mechanism.

Returning back to the $3 \times 3$ system (1.1), setting $\epsilon=0$, the three eigenvalues are

$$
\lambda_{1}=-\sqrt{\sigma_{v}(v, s)}, \quad \lambda_{2}=c_{1}, \quad \lambda_{3}=\sqrt{\sigma_{v}(v, s)} .
$$

If $\sigma_{v}(v, s) \geq 0$, then (1.1) is a hyperbolic system. For the particular cases of (1.1) considered in [Lu1] [Lu2], it is shown that there is a strictly convex entropy which gives the necessary $L^{2}$ estimates and also compactness using compensated compactness. However for the case $\sigma_{v}(v, s)<0$ we do not have an entropy in the strict sense. In this paper, our main interest is to deal with this elliptic-hyperbolic case under the weaker restriction $\frac{d \sigma}{d v}(v, h(v))>0$. In order to avoid technical details throughout this paper we assume $h(v)=c v, c$ is a constant. In fact all the steps in this paper work just as well for a more general $h(v)$ which satisfies $h^{\prime}(v) \geq c>0$. This can be seen by writing the third term in (1.1) equivalently as

$$
\frac{s-h(v)}{\tau}=\frac{h^{\prime}(\alpha)\left(h^{-1}(s)-v\right)}{\tau}
$$

where $h^{-1}$ is the inverse function of $h$ and $\alpha$ takes on a value between $h^{-1}(s)$ and $v$.

\section{Viscous RElaxation Limit}

Theorem 2.1. $\quad$ part 1:

If the initial data $\left(v_{0}, u_{0}, s_{0}\right)$ are smooth functions satisfying the condition

$\left(c_{1}\right)\left|v_{0}, u_{0}, s_{0}\right|_{L^{2} \cap L^{\infty}(R)} \leq M_{1}$

$\lim _{|x| \rightarrow \pm \infty}\left(\frac{d^{i} v_{0}}{d x^{i}}, \frac{d^{i} u_{0}}{d x^{i}}, \frac{d^{i} s_{0}}{d x^{i}}\right)=(0,0,0), \quad i=0,1$,

and $h(v)=c v, \sigma(v, s)$ satisfies the condition

$\left(c_{2}\right)\left|\sigma_{s}(v, s)\right| \leq M_{2} \quad, \bar{\sigma}^{\prime}(v) \geq d>\max \left\{0, c^{2}-c+\frac{2 c^{2} c_{1}^{2}}{\left(M_{2}+1\right)^{2}}\right\}, \quad$ where $\bar{\sigma}(v)=\sigma(v, c v)$, 
then for fixed $\epsilon, \tau$ satisfying $\tau\left(M_{2}+1\right)^{2} \leq \epsilon$, the solutions $(u, v, s) \in C^{2}$ of the Cauchy problem (1.1), (1.2) exist in $(-\infty, \infty) \times[0, T]$ for any given $T>0$ and satisfy

$$
\begin{array}{r}
|v(x, t)|, \quad|u(x, t), \quad| s(x, t) \mid \leq M(\epsilon, \tau, T), \\
\left|u^{2}(., t)\right|_{L^{1}(R)}, \quad\left|v^{2}(., t)\right|_{L^{1}(R)}, \quad\left|s^{2}(., t)\right|_{L^{1}(R)} \leq M, \\
\left|(s-c v)^{2}\right|_{L^{1}\left(R \times R^{+}\right)} \leq \tau M, \\
\left|\epsilon u_{x}^{2}\right|_{L^{1}\left(R \times R^{+}\right)},\left|\epsilon v_{x}^{2}\right|_{L^{1}\left(R \times R^{+}\right)},\left|\epsilon s_{x}^{2}\right|_{L^{1}\left(R \times R^{+}\right)} \leq M .
\end{array}
$$

- part 2:

If $\bar{\sigma}(v)=\sigma(v, c v)$ satisfies the condition

$\left(c_{3}\right) \bar{\sigma}\left(v_{0}\right)=0$ and $\bar{\sigma}^{\prime \prime}(v) \neq 0$ for $v \neq v_{0}, \bar{\sigma}^{\prime \prime}, \bar{\sigma}^{\prime \prime \prime} \in L^{2} \cap L^{\infty}$,

then there exists a subsequence $\left(v^{\epsilon, \tau}, u^{\epsilon, \tau}, s^{\epsilon, \tau}\right)$ of the solutions to the Cauchy problem (1.1), (1.2) and there exists $L^{2}$ bounded functions $(\bar{v}, \bar{u}, \bar{s})$ such that

$$
\left(v^{\epsilon, \tau}, u^{\epsilon, \tau}, s^{\epsilon, \tau}\right) \rightarrow(\bar{v}, \bar{u}, \bar{s}) \quad \text { a.e. }(x, t)
$$

as $(\epsilon, \tau) \rightarrow(0,0)$ subject to the condition $\tau\left(M_{2}+1\right)^{2} \leq \epsilon$, and $(\bar{v}, \bar{u})$ is an entropy solution of the equilibrium system (1.3) with the initial data $\left(v_{0}(x), u_{0}(x)\right)$.

Proof. To prove part 1, we use the following local existence lemma and the $L^{\infty}$ estimates given in (2.1).

Lemma 2.2 (Local existence). If the initial data satisfies the condition $\left(c_{1}\right)$ in Theorem [2.1, then for any fixed $\epsilon$ and $\tau>0$ the Cauchy problem (1.1), (1.2) admits a unique smooth local solution $(u, v, s)$ which satisfies

$$
\left|\frac{\partial^{i} v}{\partial x^{i}}\right|+\left|\frac{\partial^{i} u}{\partial x^{i}}\right|+\left|\frac{\partial^{i} s}{\partial x^{i}}\right| \leq M\left(t_{1}, \epsilon, \tau\right)<+\infty, \quad i=0,1,2,
$$

where $M\left(t_{1}, \epsilon, \tau\right)$ is a positive constant that depends only on $t_{1}, \epsilon, \tau$ and $t_{1}$ depends on $\left|v_{0}\right|_{L^{\infty}},\left|u_{0}\right|_{L^{\infty}},\left|s_{0}\right|_{L^{\infty}}$.

Moreover

$$
\lim _{|x| \rightarrow \pm \infty}\left(\frac{d^{i} v}{d x^{i}}, \frac{d^{i} u}{d x^{i}}, \frac{d^{i} s}{d x^{i}}\right)=(0,0,0), \quad i=0,1,
$$

uniformly in $t \in\left[0, t_{1}\right]$.

The proof of Lemma 2.2 is standard.

To derive the crucial estimates given in (2.1), we need the necessary condition $\tau\left(M_{2}+1\right)^{2} \leq \epsilon$ and condition $\left(c_{2}\right)$ in the Theorem 2.1

Multiply $\bar{\sigma}(v)+c v-c s$ to the first equation in (1.1), multiply $u$ to the second and $s-c v$ to the third equation and add the results. This gives

$$
\begin{aligned}
\left(\int_{0}^{v} \bar{\sigma}(v)+c v d v+\frac{u^{2}}{2}-c s v+\frac{s^{2}}{2}\right)_{t}+\left(c u s-u(\bar{\sigma}(v)+c v)+\frac{c_{1} s^{2}}{2}\right)_{x} \\
-c c_{1} v s_{x}-u(\sigma(v, s)+s-(\sigma(v, c v)+c v))_{x}+\frac{(s-c v)^{2}}{\tau} \\
=\epsilon\left(\int_{0}^{v} \bar{\sigma}(v)+c v d v+\frac{u^{2}}{2}-c s v+\frac{s^{2}}{2}\right)_{x x} \\
-\epsilon\left(\bar{\sigma}^{\prime}(v)+c\right) v_{x}^{2}-\epsilon u_{x}^{2}-\epsilon s_{x}^{2}+2 c \epsilon s_{x} v_{x} .
\end{aligned}
$$


For the third and fourth terms on the left-hand side of (2.7), we have the estimate

$$
\begin{array}{r}
-c c_{1} v s_{x}-u(\sigma(v, s)+s-(\sigma(v, c v)+c v))_{x} \\
=\left(\frac{c_{1} c^{2}}{2} v^{2}-c c_{1} v s\right)_{x}-(u(\sigma(v, s)+s-(\sigma(v, c v)+c v)))_{x} \\
+u_{x}\left(\sigma_{s}(v, \alpha)+1\right)(s-c v)+c c_{1} v_{x}(s-c v)
\end{array}
$$

where $\alpha$ takes a value between $s$ and $c v$. The last two terms in (2.8) have the upper bound

$$
\frac{3(s-c v)^{2}}{4 \tau}+\frac{\tau\left(M_{2}+1\right)^{2} u_{x}^{2}}{2}+\tau c^{2} c_{1}^{2} v_{x}^{2}
$$

by the first condition in $\left(c_{2}\right)$.

Combining (2.7), (2.8) and (2.9), we get the following inequality:

$$
\begin{array}{r}
\left(\int_{0}^{v} \bar{\sigma}(v)+c v d v+\frac{u^{2}}{2}-c s v+\frac{s^{2}}{2}\right)_{t}+\left(c u s-u(\bar{\sigma}(v)+c v)+\frac{c_{1}}{2} s^{2}\right)_{x} \\
\left(c c_{1} v s-\frac{c_{1} c^{2}}{2} v^{2}\right)_{x}-(u(\sigma(v, s)+s-(\sigma(v, c v)+c)))_{x}+\frac{(s-c v)^{2}}{4 \tau} \\
\leq \epsilon\left(\int_{0}^{v} \bar{\sigma}(v)+c v d v+\frac{u^{2}}{2}-c s v+\frac{s^{2}}{2}\right)_{x x}-\epsilon\left(\bar{\sigma}^{\prime}(v)+c\right) v_{x}^{2}-\epsilon s_{x}^{2}+2 c \epsilon s_{x} v_{x} \\
+c^{2} c_{1}^{2} \tau v_{x}^{2}-\left(\epsilon-\frac{\tau\left(M_{2}+1\right)^{2}}{2}\right) u_{x}^{2}
\end{array}
$$

Noticing the second condition in $\left(c_{2}\right)$ we know that $\left(\int_{0}^{v} \bar{\sigma}(v)+c v d v+\frac{u^{2}}{2}-c s v+\right.$ $\left.\frac{s^{2}}{2}\right)$ is a strictly convex function. If the condition $\tau\left(M_{2}+1\right)^{2} \leq \epsilon$ is satisfied, we immediately get the estimates (2.2), (2.3) by integrating (2.10) on $R \times[0, T]$ with the behaviour in (2.6). Differentiating the first equation in (1.1) with respect to $x$, we get

$$
\left(v_{x}\right)_{t}-u_{x x}=\epsilon\left(v_{x}\right)_{x x}
$$

Multiplying $v_{x}$ to (2.11) yields

$$
\left(\frac{v_{x}^{2}}{2}\right)_{t}-\left(v_{x} u_{x}\right)_{x}+u_{x} v_{x x}=\epsilon\left(\frac{v_{x}^{2}}{2}\right)_{x x}-\epsilon v_{x x}^{2} .
$$

Integrating (2.12) on $R \times[0, T]$ and noticing the bound $\left|u_{x}^{2}\right|_{L^{1}\left(R \times R^{+}\right)} \leq M(\epsilon)$, we obtain the bound $\left|v_{x}^{2}(., t)\right|_{L^{1}(R)} \leq M(\epsilon)$, where $M(\epsilon)$ is a constant depending on $\epsilon$. Therefore

$$
v^{2}=\left|\int_{-\infty}^{x}\left(v^{2}\right)_{x} d x\right| \leq \int_{-\infty}^{\infty} v^{2} d x+\int_{-\infty}^{\infty} v_{x}^{2} d x \leq M(\epsilon)
$$

Similarily from the second and third equations in (1.1) we get $\left|s_{x}^{2}(., t)\right|_{L^{1}(R)} \leq M(\epsilon)$ and $\left|u_{x}^{2}(., t)\right|_{L^{1}(R)} \leq M(\epsilon)$. So we get the estimates in (2.1) and the proof of part 1 in Theorem 2.1.

From the estimates in (2.2) and (2.3) it is easy to prove the compactness of $\eta\left(v^{\epsilon, \tau}\right)_{t}+q\left(v^{\epsilon, \tau}\right)_{x}$ in $H_{l o c}^{-1}\left(R \times R^{+}\right)$, where $\left(v^{\epsilon, \tau}, u^{\epsilon, \tau}\right)$ are the solutions of the Cauchy problem (1.1), (1.2) and $(\eta, q)$ is any entropy-entropy flux pair constructed in [S]. Using the technique in $[\mathrm{S}]$ the convergence of $\left(v^{\epsilon, \tau}, u^{\epsilon, \tau}\right)$ can be obtained. For 
details see [Lu1], Lu2]. From the first estimate in (2.3) we obtain the convergence $s^{\epsilon, \tau} \rightarrow \bar{s}$. So Theorem 2.1 is proven.

\section{ACKNOWLEDGEMENT}

Yun-guang Lu's research was partially supported by a Humboldt's fellowship, a research project from the National University of Colombia, Bogota and NNSF 10071080 of China.

\section{REFERENCES}

[D] R.J. DiPerna, Convergence of approximate solutions to conservation laws, Arch. Rational Mech. Anal., 82(1983), 27-70. MR 84k:35091

[KL] C. Klingenberg, Y.G. Lu, Cauchy problem for Hyperbolic conservation laws with a relaxation term, Proc. Roy. Soc. of Edinb., Series A, 126(1996), 821-828. MR 97f:35130

[Liu] T.P. Liu, Hyperbolic Conservation Laws with Relaxation, Comm. Math. Phys., 108(1987), 153-175. MR 88f:35092

[L1] Y.G. Lu, Cauchy problem for an extended model of combustion, Proc. Roy. Soc. Edin., 120A(1992), 349-360. MR 93f:80021

[L2] Y.G. Lu, Cauchy problem for a hyperbolic model, Nonlinear Analysis, TMA, 23(1994), 1135-1144. MR 95i:35177

[L3] Y.G. Lu, Singular limits of stiff relaxation and dominant diffusion for nonlinear systems, J. Diff. Equs., 179(2002), 687-713. MR 2003b:35134

[Lu1] Y.G. Lu and C. Klingenberg, The Cauchy problem for hyperbolic conservation laws with three equations, J. Math. Appl.Anal., 202(1996), 206-216. MR 97h:35147

[Lu2] Y.G. Lu and C. Klingenberg, The relaxation limit for systems of Broadwell type, Diff. Int. Equations, 14(2001), 117-127. MR 2001k:35200

[M] A. Majda, A qualitative model for dynamic combustion, SIAM J. Appl. Math., 41(1981), 70-93. MR 82j:35096

[S] J. Shearer, Global existence and compactness in $L^{p}$ for the quasilinear wave equation, Comm. PDE, 19(1994), 1829-1877. MR 95m:35120

[T] A. Tzavaras, Materials with internal variables and relaxation to conservation laws, Arch. Rational Mech., 146 (1999), 129-155. MR 2000i:74004

Department of Mathematics, University of Science and Technology of China, Hefei, People's Republic of China - and - Departamento de Matemáticas, Universidad Nacional de Colombia, Bogotá, Colombia

E-mail address: yglu@matematicas.unal.edu.co

Applied Mathematics, Würzburg University, Am Hubland, Würzburg 97074, Germany

E-mail address: Christian.Klingenberg@iwr.uni-heidelberg.de 